$\frac{7}{202911}$ the

PPPL-2786

UC-420, 427
PREPARED FOR THE U.S. DEPARTMENT OF ENERGY, UNDER CONTRACT DE-AC02-76-CHO-3073

PPPL-2786

CURRENT-DRIVE BY LOWER HYBRID WAVES IN

THE PRESENCE OF ENERGETIC ALPHA-PARTICLES

BY

N.J. FISCH AND J-M RAX

October 1991

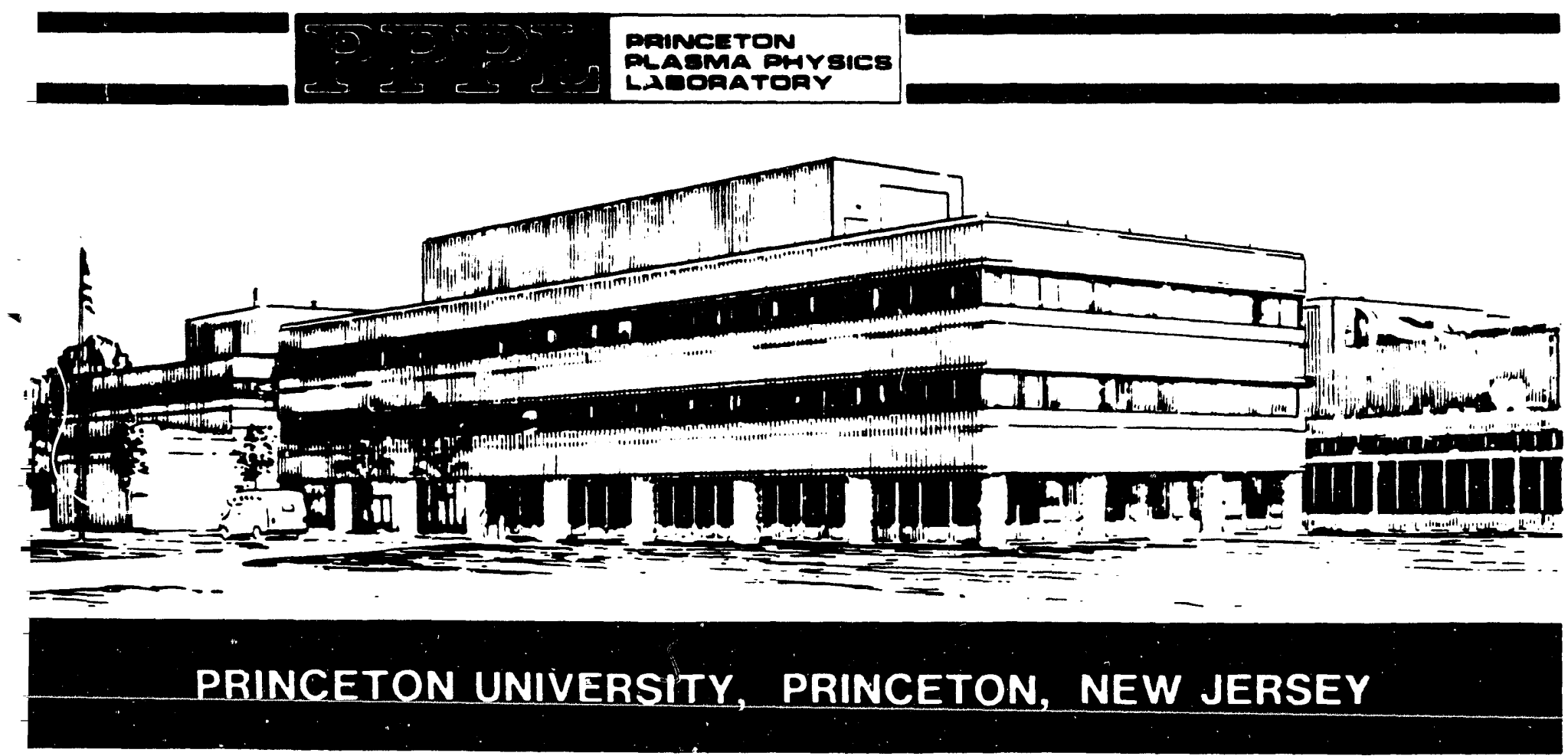




\section{NOTICE}

This report was prepared as an account of work sponsored by an agency of the United States Government. Neither the United States Government nor any agency thereof, nor any of their employees, makes any warranty, express or implied, or assumes any legal liability or responsibility for the accuracy, completeness, or usefulness of any information, apparatus, product, or process disclosed, or represents that its use would not infringe privately owned rights. Reference herein to any specific commercial produce, process, or service by trade name, trademark, manufacturer, or otherwise, does not necessarily constitute or imply its endorsement, recommendation, or favoring by the United States Government or any agency thereof. The views and opinions of authors expressed herein do not necessarily state or reflect those of the United States Government or any agency thereof.

\section{NOTICE}

This report has been reproduced directly from the best available copy.

Available to DOE and DOE contractors from the:

Office of Scientific and Technical Information

P.O. Box 62

Oak Ridge, TN 37831;

Prices available from (615) 576-8401.

Available to the public from the:

National Technical Information Service

U.S. Department of Commerce

5285 Port Royal Road

Springfieid, Virginia 22161

$703-487-4650$ 


\title{
Current-drive by Lower Hybrid Waves in De92 001940 the Presence of Energetic Alpha-Particles
}

\author{
Nathaniel J. Fisch and Jean-Marcel Rax ${ }^{\dagger}$ \\ Princeton Plasma Physics Laboratory, Princeton University, Princeton, NJ 08543
}

\begin{abstract}
Many experiments have now proved the effectiveness of lower hybrid waves for driving toroidal current in tokamaks. The use of these waves, however, to provide all the current in a reactor is thought to be uncertain because the waves may not penetrate the center of the more energetic reactor plasina, and, if they did, the wave power may be absorbed by alpha particles rather than by electrons. This paper explores the conditions under which lower-hybrid waves might actually drive all the current.
\end{abstract}

$\dagger$ permanent address: Centre d'études nucléaires de Cadarache, Association EURATOM-CEA sur la fusion contrôlée, 13108 Saint-Paul-iez-Durance, France. 


\section{Introduction}

Lower hybrid waves can be effective and efficient for driving toroidal current in tokamaks, ${ }^{1}$ and the central aspects of the theory have now been established by many experiments. ${ }^{2-5}$ There would be a great benefit if these waves could be employed to provide the full toroidal tokamak current, thereby allow ing tokamak reactors to operate in the steady state. At present, over two MA of current are routinely produced by lower hybrid waves in laboratory tokamaks, yet the use of these waves to provide all the current in a reactor is still uncertain. The problems in extrapolating the results relate to the physics of the very center of a reactor tokamak: first, the waves may not penetrate the center of the more energetic reactor plasma, and, second, if they did, as pointed out first by Wong and $\mathrm{Ono}^{6}$, the wave power may be absorbed by $\alpha$-particles rather than by electrons.

Avoidance of the damping by $\alpha$-particles can be accomplished only by using waves of high frequency. These higher frequency waves, however, are technically more difficult to inject into the plasma; the coupling to the plasma may be less efficient; and, if waveguides are employed, the structural dimensions will be smaller at high frequency and more difficult to fabricate. Recent calculations by Bonoli and Porkolab, ${ }^{7}$ Barbato and Santini, ${ }^{8}$ and by Spada et al. ${ }^{9}$ explore the possibilities for avoiding the damping by $\alpha$-particles. The calculations of Barbato and Santini include the quasilinear effect of the waves on the $\alpha$ particle distribution.

The question of $\alpha$-particle damping would be moot, however, if the waves do not penetrate the plasma center. Penetration of the plasma center is not likely to occur in the dense, hot, reactor plasma, because there are sufficiently many hot electrons at a distance from the plasma center that absorb the lower hybrid power. In this case, the lower-hybrid driven current appears, but it would have a hollow profile. Hollow current profiles are not thought to be stable, so the injection of such a current would not be suitable for maintaining the complete plasma current.

A number of detailed reactor stuaies reach the conclusion that, in order to provide the full current in a reactor, lower-hybrid current drive will need to be supplemented by other means of current drive. (Such a design is pursued in the current plans for ITER. ${ }^{10}$ ) These conclusions are reached precisely because of the assumption that the current appears only on the magnetic surface in which the wave power is absorbed. The experimental evidence for this assumption is not compelling; in fact, if anything, the evidence is counter-indicating. Because the supplementary means of current drive are an undesirable complicating feature in react $r$ design, this assumption leads to modeling efforts that may be too conservative, and to a design for a much less desirable reactor. It is worthwhile, therefore, to examine this key assumption in depth before designing on the basis of it.

It may be the case that even if the waves are damped far from the plasma, the current might diffuse to the center. This decoupling of current profile from deposition profile would mean that the waves could drive a central current without penetrating the center. While this suggestion, with monumental implications, is speculative, the possibility that the current-carrier profile closely follows the deposition profile must be viewed with at least equal skepticism. Until experiments clarify the matter, the relation between power deposition and current profile will remain an open question.

If the current could be provided through the deposition of power away from the plasma 
center, then a number of favorable circumstances appear related to the interaction with $\alpha$-particiss: The energetic alpha particles, produced primarily in the plasma center, are fewer and less energetic off center. The lower hybrid waves, on the other hand, have a decreasing phase velocity as the waves enter the higher density plasma center, so the $\alpha$ particle damping is much less off-center than at the plasma center. Moreover, while an isotropic distribution of $\alpha$-particles will always damp the lower-hybrid waves (so long as the energetic particles can be considered unmagnetized in the wave fields), this assertion fails if the distribution function is not isotropic. In fact, as the $\alpha$-particles both slow down and diffuse away from where they are produced (near the plasma center), their distribution could become anisotropic off-center. Since the $\alpha$-particle distribution is energy inverted, the possiblity exists then that, off-center, the $\alpha$-particles might even amplify the lowerhybrid waves.

The following possibility therefore emerges as a very favorable set of circumstances for lower-hybrid current drive: the lower-hybrid waves damp on electrons, primarily offcenter, perhaps only half-way towards the magnetic axis; the current-carrying fast electrons produced by the lower-hybrid waves diffuse to the plasma center, thereby producing a normal current profile without the need for auxiliary current drive apparatus; and the $\alpha$-particles, diffusing out of the plasma center, but slowing down, possibly anisotropically, do not significantly damp the lower-hybrid waves off-center, and possibly provide some small amplification. While, to be sure, this favorable scenario is uncertain, neither can it be assumed, on the basis of current data, that it is incorrect.

The speculation that lower-hybrid waves might provide the full current even in the presence of fusion-generated $\alpha$-particles can be analyzed through a consideration of the details of the wave damping on $\alpha$-particles, the evolution of the $\alpha$-particie distribution function including quasilinear velocity diffusion and spatial diffusion, and the spatial diffusion of energetic current-carrying electrons. In this paper, we attempt, in light of the goal of generation by if waves of the full current, to assess and advance our understanding of each of these issues.

The paper is organized as follows: In Sec. 2 we derive the one-dimensional $\alpha$-particle distribution function under the influence of intense of diffusion, but no spatial diffusion. In Sec. 3 we derive the powrer dissipation based on the 1-D velocity distribution. The very interesting finding of Secs. 2 and 3 is that an exact one dimensional distribution function can be found analytically, and that the exact power dissipation can be written in terms of this distribution function. We find a quasilinear damping law for $\alpha$-particle damping at high of power that scales with the $4 / 5$ power of the lower-hybrid wave spectral intensity, while for the electron damping, there is saturation at high power ("0 power" law scaling). Of practical interest is that, as other authors have found, the resonant interaction of the lower-hybrid waves with the $\alpha$-particles ought to be avoided altogether. In Sec. 4 we derive the one-dimensional $\alpha$-particle distribution function dissipation in the presence of $\boldsymbol{\alpha}$-particle diffusion in configuration space, but in the absence of resonant wave-particle interactions. In Sec. 5 we explore the empirical evidence for nonlocal current drive. In Sec. 6 we present our conclusions and suggestions for experimentation to decide key issues. 


\section{Fokker-Planck Equation}

Energetic alpha particles collide primarily with electrons, so the slowing down of an $\alpha$-particle with velocity $\mathbf{v}$ obeys

$$
\frac{d \mathbf{v}}{d t}=-\nu \mathbf{v}
$$

where the collision frequency $\nu=16 \sqrt{2 \pi m_{e}} e^{4} n_{e} \ln \Lambda / 3 T_{e}^{3 / 2} m_{\alpha}$. For a distribution of $\alpha$ particles, we then have a kinetic equation

$$
\frac{\partial}{\partial t} f(\mathbf{v}, t)=-\frac{\partial}{\partial \mathbf{v}} \cdot \mathbf{S}+\frac{\dot{N}}{4 \pi v_{\alpha}^{2}} \delta\left(v-v_{\alpha}\right)
$$

where the second term describes the birth of $\alpha$-particles at $3.5 \mathrm{MeV}$ at a rate $\dot{N}$ and the velocity-space flux $\mathbf{S}$ is given by

$$
\mathbf{S}=-\nu \mathbf{v} f-\mathbf{D}_{\mathbf{r f}} \cdot \frac{\partial}{\partial \mathbf{v}} f
$$

where the first term describes the slowing down of $\alpha$-particles in collisions with electrons and where $D_{r f}$ is the quasilinear diffusion coefficient.

For a Landau resonant interaction, with $k_{\perp} \gg k_{\|}$, the diffusion is essentially in the perpendicular direction, and the magnitude of the quasilinear diffusion coefficient can be written, correctly for unmagnetized $\alpha$-particles, ${ }^{11}$ as

$$
\mathrm{D}_{\mathrm{rf}}=D_{Q L}\left(v_{\perp}\right) \hat{\imath}_{\perp} \hat{\imath}_{\perp}
$$

with

$$
D_{Q L}= \begin{cases}0, & \text { if } v_{\perp}<\omega / k_{\perp} \\ \frac{1}{2}\left(e Z_{\alpha} E / m_{\alpha}\right)^{2}\left(\omega / k_{\perp} v_{\perp}\right)^{2}\left(k_{\perp}^{2} v_{\perp}^{2}-\omega^{2}\right)^{-1 / 2}, & \text { if } v_{\perp}>\omega / k_{\perp},\end{cases}
$$

where $E$ is the electric field of the lower hybrid wave, $\omega$ is the wave frequency, $k_{\perp}$ is the wave perpendicular wavenumber, and $Z_{\alpha}=2$ is the $\alpha$-particle charge state.

It is quite remarkarkable that he problem is then inherently one-dimensional. In contrast, other so-called 1-D problems, such as current-drive by electron Landau-damping, are really just approximations. The problem of $\alpha$-particle damping is rigorously onedimensional, because the collisions of $\alpha$-particles with electrons results in a contraction of the $\alpha$-particle distribution function in parallel velocity, and this contraction affects neither the rate of slowing down nor the wave-absorption resonance. This can be seen as follows. Let us write

$$
-\frac{\partial}{\partial \mathbf{v}} \cdot \nu \mathbf{v} f=-\left(\hat{\imath}_{\perp} \frac{1}{v_{\perp}} \frac{\partial}{\partial v_{\perp}} v_{\perp}+\hat{\imath}_{\|} \frac{\partial}{\partial v_{\|}}\right) \cdot \mathbf{v} f=-\left(\frac{1}{v_{\perp}} \frac{\partial}{\partial v_{\perp}} v_{\perp}^{2} f+\frac{\partial}{\partial v_{\|}} v_{\|} f\right)
$$

Define

$$
F\left(v_{\perp}, t\right) \equiv \int_{-\infty}^{+\infty} f(\mathbf{v}, t) d v_{\|}
$$


Then integrating Eq.(2) over $v_{\|}$we get

$$
\frac{\partial}{\partial t} F\left(v_{\perp}, t\right)=-\frac{1}{v_{\perp}} \frac{\partial}{\partial v_{\perp}} v_{\perp}\left(S_{\perp}\left(v_{\perp}, t\right)+Q_{\alpha}\right)
$$

where the $\alpha$-particle source flux, $Q_{\alpha}$, is then

$$
Q_{\alpha}=H\left(v_{\alpha}-v_{\perp}\right) \frac{1}{v_{\perp}} \frac{\dot{N}}{2 \pi v_{\alpha}} \sqrt{v_{\alpha}^{2}-v_{\perp}^{2}}
$$

where $H$ is a Heaviside function $(H(x)=1, x>0$ else $H(x)=0$ ), and the perpendicular flux, $S_{\perp}\left(v_{\perp}, t\right)$, is given by

$$
S_{\perp}\left(v_{\perp}, t\right)=-\nu v_{\perp} F\left(v_{\perp}, t\right)-D_{Q L} \frac{\partial}{\partial v_{\perp}} F\left(v_{\perp}, t\right) .
$$

In general, supplementing Eq.(8) by an initial condition for $F\left(v_{\perp}, t=0\right)$ and by boundary conditions, for example, at $v_{\perp}=v_{0}$, where $v_{0}$ is some finite speed, and at $v_{\perp} \rightarrow \infty$ results in a well-posed diffusion equation. Here, the physical boundary conditions of interest are for $\alpha$-particles that slow down into a relatively cold thermal distribution, which we treat as a sink. In other words, we impose a perfectly reflecting (zero flux) boundary at $v_{\perp} \rightarrow \infty$ and a perfectly absorbing boundary at $v_{\perp} \rightarrow 0$. The first condition is realized if $F$ or $S_{\perp}$ vanish at $v_{\perp} \rightarrow \infty$. The absorption condition as $v_{\perp} \rightarrow 0$ can be realized by matching the full flux to the collisional flux $-\nu v_{\perp} F\left(v_{\perp}, t\right)$. In other words, particles escape the region of interest to lower $v_{\perp}$ at a rate dictated just by slowing down collisions.

For the interesting case of steady state, the flux $S_{\perp}$ must vanish in the sourceless, semi-infinite region $v_{\perp}>v_{\alpha}$. In the region $v_{\perp}<v_{\alpha}$, we can integrate Eq.(8) to get

$$
\nu v_{\perp} F\left(v_{\perp}\right)+D_{Q L} \frac{\partial}{\partial v_{\perp}} F\left(v_{\perp}\right)=\frac{1}{v_{\perp}} \frac{\dot{N}}{2 \pi v_{\alpha}} \sqrt{v_{\alpha}^{2}-v_{\perp}^{2}}
$$

where the constant of integration is zero since the flux must vanish at $v_{\perp}=v_{\alpha}$. Note that as $v_{\perp} \rightarrow 0,2 \pi v_{\perp} S_{\perp} \rightarrow \dot{N}$, since there is presumed to be a sink at $v_{\perp}=0$ to collect, in the steady state, the complete $\alpha$-particle production.

Eq.(10) is a first-order ordinary differential equation, and so it is readily solved exactly. Let us introduce the nondimensional variables, $s=v_{\perp} / v_{\alpha}, \beta=\omega / k_{\perp} v_{\alpha}$, and $G=F /\left(\dot{N} / 2 \pi \nu v_{\alpha}^{2}\right)$. Using the quasilinear diffusion coefficient in Eq.(5), Eq.(10) may then be cast in the form

$$
D H(s-\beta)\left(\frac{1}{s^{2}-\beta^{2}}\right)^{1 / 2} \frac{d G(s)}{d s}+s^{3} G(s)=s \sqrt{1-s^{2}} H(1-s),
$$

where we defined $D$, a normalized measure of diffusion, as

$$
D \equiv \frac{1}{2}\left(\frac{e Z_{\alpha} E}{m}\right)^{2} \frac{\beta^{3}}{\nu \omega v_{\alpha}^{2}}=\frac{D_{Q L}}{\nu v_{\alpha}^{2}} s^{2} \sqrt{s^{2}-\beta^{2}} .
$$


For $\omega / k_{\perp}>0$, both the particle source and the diffusion vanish at the boundary with the collisional region, $v_{\perp} \rightarrow 0$, so that boundary condition can be realized by matching the solution of Eq.(11) for $s>\beta$ to the solution valid for $s<\beta$, namely

$$
G(s)=s^{-2} \sqrt{1-s^{2}}, \quad s<\beta .
$$

Let us define for $s>\beta$

$$
\Psi(s) \equiv \frac{1}{D} \int_{\beta}^{s} x^{3} \sqrt{x^{2}-\beta^{2}} d x=\frac{1}{5 D}\left(s^{2}-\beta^{2}\right)^{3 / 2}\left(s^{2}+2 \beta^{2} / 3\right),
$$

then the solution to Eq.(11) can be written for $s>\beta$ as

$$
G(s)=\mathrm{e}^{-\Psi(s)}\left[\frac{\sqrt{1-\beta^{2}}}{\beta^{2}}+\frac{1}{D} \int_{\beta}^{s} x \mathrm{e}^{\Psi(x)} \sqrt{x^{2}-\beta^{2}} \sqrt{1-x^{2}} H(1-x) d x\right] .
$$

We note that the integrating factor can be expressed in terms of elementary functions, as done on the right-hand side of Eq.(14), but further analytic progress appears possible only in certain asymptotic limits, which may be of physical interest and are described in the following section.

\section{Power Dissipated}

The power dissipated by the waves turns out to be available exactly in terms of the one-dimensional solution. Since the rf-induced flux is perpendicular, we can write the wave power dissipated as

$$
P_{r f}=\int d^{3} v m \mathbf{v} \cdot \mathbf{S}_{\mathrm{rf}}=\int 2 \pi v_{\perp} d v_{\perp} m v_{\perp} \int d v_{\|} \mathbf{S}_{\mathrm{rf}}=\int 2 \pi v_{\perp} d v_{\perp} m v_{\perp} D_{Q L} \frac{\partial F}{\partial v_{\perp}}
$$

where we carried out the integration over $v_{\|}$trivially, since $\mathbf{S}_{\mathrm{rf}}$ is independent of $v_{\|}$. This power can be contrasted to the $\alpha$-particle heating power, which can be written as

$$
P_{\alpha}=\dot{N} m v_{\alpha}^{2} / 2 \text {. }
$$

Consider the normalized wave power (where a negative number indicates wave damping)

$$
P_{w} \equiv \frac{P_{r f}}{P_{\alpha}}=2 D \int_{\beta}^{\infty}\left(s^{2}-\beta^{2}\right)^{-1 / 2} \frac{d G}{d s} d s=2 \int_{\beta}^{\infty} d s\left[s \sqrt{1-s^{2}} H(1-s)-s^{3} G\right],
$$

where in writing the first equality we used Eqs.(10), (11) and (12), and in writing the second equality we used Eq.(11). The correct power dissipated may be found now by use of Eq.(15). Note that the calculation of this power dissipated is exact for the physical model at hand - namely, that $\alpha$ particles are subject to slowing-down collisions only and that the rf power results in perpendicular diffusion only. There are no approximations introduced 
through our mathematical use of the one-dimensional perpendicular distribution function. To calculate accurately the fully two-dimensional distribution function $f\left(v_{\perp}, v_{\|}\right)$is a more difficult calulation, and might be approached approximately, for example, by an expansion in Legendre harmonics. ${ }^{8}$ The fully two-dimensional distribution function $f\left(v_{\perp}, v_{\|}\right)$ is sufficient but not necessary to calculate the dissipated power, but an approximated two-dimensional distribution function will only give an approximated dissipated power; here, the one-dimensional distribution function $F\left(v_{\perp}\right)$, which is available exactly, not only suffices, but is guaranteed to give the exact power dissipation.

Note, however, that this simplification would not be possible if the pitch-angle scattering of the $\alpha$-particles were important. This limits our consideration to wave phase velocities far greater than about $500 \mathrm{keV}$, which is where siowing down and pitch angle scattering are about equal. In practice, this limit is of little concern, since the waves must avoid entirely the less energetic $\alpha$-particles.

The power dissipated can be estimated analytically in a number of interesting limits. If $\beta>1$, then, of course, no power is dissipated on the $\alpha$-particles. Of great interest, however, is how sensitive the dissipation is under high power to a relaxation of the condition that $\beta>1$; in other words, can some $\alpha$-particles be resonant with the wave, resulting in tolerable dissipation with $\beta \leq 1$. Consider the limit $\beta \rightarrow 1$; then most of the wave power is dissipated through resonant interactions in the region $s>1$, namely through acceleration of $\alpha$-particles to speeds greater than their birth speed. This can be seen as follows: The first term on the right-hand side of Eq.(18), representing interactions in the regime $s<1$ can be calculated directly as

$$
\int_{\beta}^{\infty} s \sqrt{1-s^{2}} H(1-s) d s=\frac{1}{2} \int_{\beta^{2}}^{1} \sqrt{1-x} d x=\frac{1}{3}\left(1-\beta^{2}\right)^{3 / 2} .
$$

This integral can be compared to the second term on the right-hand side of Eq.(18), which, by using Eq.(15), we can write as the sum of two terms, namely

$$
-\int_{\beta}^{\infty} s^{3} G d s=-\int_{\beta}^{\infty} s^{3} \mathrm{e}^{-\Psi(s)}\left[\frac{\sqrt{1-\beta^{2}}}{\beta^{2}}+\frac{1}{D} \ldots\right] \equiv \mathrm{A}+\mathrm{B} .
$$

Term $A$ can be put in the form

$$
\mathrm{A} \equiv-\frac{\sqrt{1-\beta^{2}}}{\beta^{2}} \int_{\beta}^{\infty} s^{3} \mathrm{e}^{-\frac{1}{5 D}\left(s^{2}-\beta^{2}\right)^{3 / 2}\left(s^{2}+2 \beta^{2} / 3\right)} d s=-D^{4 / 5} \frac{\sqrt{1-\beta^{2}}}{2 \beta^{2}} g\left(\beta^{2} / D^{2 / 5}\right),
$$

where

$$
g(x)=\int_{x}^{\infty} y \mathrm{e}^{-\frac{1}{5}(y-x)^{3 / 2}(y+2 x / 3)} d y .
$$

Now in the limit $\beta^{2} \sim O(1)$, but $D \rightarrow \infty$, we have $x \rightarrow 0$ and we can approximate

$$
\lim _{x \rightarrow 0} g(x)=\int_{0}^{\infty} y \mathrm{e}^{-y^{-8 / 2} / 5}\left(1+\frac{x y^{3 / 2}}{6}+O\left(x^{2}\right)\right)=\frac{2 \Gamma(4 / 5)}{5^{1 / 5}}+O(x),
$$


where we used the Gamma function, $\Gamma(x) \equiv x \Gamma(x-1)$. Thus, we have for $D \rightarrow \infty$

$$
\mathrm{A} \equiv-\frac{\Gamma(4 / 5)}{5^{1 / 5}} \frac{\sqrt{1-\beta^{2}}}{\beta^{2}} D^{4 / 5}+O\left(D^{2 / 5}\right) .
$$

Note that the contribution term $A$ to the power dissipated is $D^{4 / 5}\left(1-\beta^{2}\right)^{-1}$ larger than the term, considered in Eq.(19), which represented interactions in the regime $s<1$.

Let us now show that term $B$ is negligible compared to term $A$ in the regime of interest. From Eq.(20), we have

$$
\mathrm{B} \equiv-\frac{1}{D} \int_{\beta}^{\infty} s^{3} \mathrm{e}^{-\frac{1}{5 D}\left(s^{2}-\beta^{2}\right)^{3 / 2}\left(s^{2}+2 \beta^{2} / 3\right)} I(s) d s,
$$

where, for most of the interval of interest, namely $s>1$, but $\beta \rightarrow 1$

$$
\begin{aligned}
I(s) & \equiv \int_{\beta}^{s} x \mathrm{e}^{\Psi(x)} \sqrt{x^{2}-\beta^{2}} \sqrt{1-x^{2}} H(1-x) d x=\int_{\beta}^{1} x \mathrm{e}^{\Psi(x)} \sqrt{x^{2}-\beta^{2}} \sqrt{1-x^{2}} d x \\
& \sim \int_{\beta}^{1} \sqrt{(x-\beta)(1-x)} d x \sim(1-\beta)^{2} .
\end{aligned}
$$

Thus, term $\mathrm{B}$ is smaller than term $\mathrm{A}$ for large $D$ and $\beta \rightarrow 1$ by a factor of $(1-\beta)^{-3 / 2} / D$. totically

Since term $A$ is the largest term both for large $D$ and $\beta \rightarrow 1$, it follows that asymp-

$$
P_{w} \rightarrow 1.19 D^{4 / 5} \sqrt{1-\beta}
$$

from which it follows that, in order for the rf dissipated power to be small compared to the $\alpha$-particle heating power, the lower bound of phase velocities must obey $1-\beta \sim D^{-8 / 5}$, so that for large $D, \beta$ is essentially equal to one, from which we may infer that there is essentially no leeway in the requirement that none of the $\alpha$-particles be resonant with the rf spectrum in the limit of high rf power.

This is an interesting limit, and very different from the electron interaction in the limit of high power. Under low rf power and a Landau resonant interaction, the rf power dissipated both in the $\alpha$-particles and in the electrons is proportional to the of power. For the electrons, however, under intense off excitation, there is a maximum absorbed power, and the absorbed power then scales as a constant with increasing rf power. In contrast, at high power the $\alpha$-particle absorption is proportional to the $4 / 5$ power of the rf intensity. The physical reason for this difference is clear: For a Landau resonant interaction, the magnetized electrons exhibit a quasilinear plateau, so their capability of absorbing power is limited. On the other hand, the $\alpha$-particle absorption of power is highly nonlinear in that with more if power, more $\alpha$-particles are accelerated into the region $s>\beta\left(v_{\perp}>\omega / k\right)$ where they can then be subjected to further acceleration.

\section{Wave Absorption in the Presence of Spatial Diffusion of $\alpha$-Particles}

Given the necessity of avoiding damping by $\alpha$-particles, it is important to know, in detail, the $\alpha$-particle distribution as a function of both perpendicular velocity and 
configuration space. Since nonlinear effects at high power tend to exacerbate the damping, rather than, as for electrons, alleviate the damping, resonant interactions with the $\alpha$ particles are best avoided altogether. In that case, the $\alpha$-particle distribution function, $F\left(v_{\perp}, r\right)$, can be calculated in the absence of the if interaction. The linear damping rate can then be calculated on the basis of that distribution function. On the basis of the result of the last section, it would be anticipated that, if the $\alpha$-particle source is isctropic in velocity space, quasilinear effects do not decrease the dam jing, and so the wave phase velocity needs to be faster than the fastest $\alpha$-particles to avoid damping of the wave.

The processes that determine the alpha particle distribution function $f(\mathbf{v}, \mathbf{r}, t)$ are then the energy slowing down due to the collisions with electrons, the $\alpha$-particle source, and the spatial transport. The first two processes can be described adequately, as done in writing Eq.(2), but the physics of $\alpha$-particle transport is still uncertain, both theoretically and experimentally. Suppose that the transport of $\alpha$-particles is diffusive in nature and can be described by a diffusion coefficient of the form $\mathbf{D}=\mathbf{D}(\mathbf{v})$. The kinetic equation governing the $\alpha$-particle distribution can then be written as in Eq.(2) as

$$
\frac{\partial}{\partial t} f(\mathbf{v}, \mathbf{r}, t)=-\frac{\partial}{\partial \mathbf{v}} \cdot \mathbf{S}+\frac{\dot{n}_{s}(\mathbf{r})}{4 \pi v_{\alpha}^{2}} \delta\left(v-v_{\alpha}\right)+\nabla \cdot \mathbf{D}(\mathbf{v}) \cdot \nabla f
$$

where $\dot{n}_{s}(\mathbf{r})$ is the source density, where the last term accounts for the spatial diffusion, and where the flux $\mathbf{S}$ now represents collisional flux only. For diffusion out of a cylinder, we are interested in the distribution of $\alpha$-particles as a function of the perpendicular velocity direction and as a function of radial distance $r$; hence, define

$$
F\left(v_{\perp}, r, t\right) \equiv \int_{-\infty}^{+\infty} f(\mathbf{v}, \mathbf{r}, t) d v_{\|} d z
$$

where $\hat{z}$ is the ignorable direction along the cylinder axis.

There are a number of diffusion coefficients with very different parametric dependencies that have been proposed for $\alpha$-particle transport, ${ }^{12-13}$ and certain toroidal effects might occur even in the wave-particle interaction. ${ }^{14}$ The general case will be hard to consider, but some insight may be gained by studyiong a simpler, analytically tractable problem. Let us, for simplicity, restrict our attention to a cylindrical geometry, and, further, to a diagonal diffusion coefficient, such that the radial component is a function of perpendicular velocity only, i.e., of the form $\mathbf{D}(\mathbf{v})=D_{r}\left(v_{\perp}\right) \hat{r} \hat{r}+D_{z}(\mathbf{v}) \hat{z} \hat{z}$. (Such a diffusion might arise, for example, if the typical radial step size in a collision were the $\alpha$-particle gyroradius.) Then, we can integrate nver Eq.(28) to derive

$$
\frac{\partial}{\partial t} F\left(v_{\perp}, r, t\right)=\frac{\nu}{v_{\perp}} \frac{\partial}{\partial v_{\perp}} v_{\perp}^{2} F+\frac{D_{r}\left(v_{\perp}\right)}{r} \frac{\partial}{\partial r} r \frac{\partial}{\partial r} F+\frac{\dot{n}(r)}{2 \pi v_{\alpha}}\left(v_{\alpha}^{2}-v_{\perp}^{2}\right)^{-1 / 2} H\left(v_{\alpha}-v_{\perp}\right)
$$

where $\dot{n}(r)$ is the axially integrated source density, i.e.,

$$
\dot{n}(r) \equiv \int d z \dot{n}_{s}(\mathbf{r})
$$


In the steady state, $\partial / \partial t=0$, we can cast Eq.(30) into an inhomogeneous diffusion equation for $\Psi=v_{\perp}^{2} F$. Using the new variable $u$,

$$
u\left(v_{\perp}\right) \equiv-\int_{0}^{v_{\perp}} \frac{D(s)}{\nu s} d s
$$

we can write

$$
\frac{\partial}{\partial u} \Psi(u, r, t)=\frac{1}{r} \frac{\partial}{\partial r} r \frac{\partial}{\partial r} \Psi+S(u) \dot{n}(r)
$$

where we defined

$$
S(u)=\frac{v_{\perp}^{2}}{2 \pi v_{\alpha} D\left(v_{\perp}\right)}\left(v_{\alpha}^{2}-v_{\perp}^{2}\right)^{-1 / 2} .
$$

Eq.(32) is an inhomogeneous diffusion equation with constant coefficient, in cylindrical geometry, to be solved with homogeneous initial and boundary conditions. It has the well-known Green's function solution

$$
G\left(u-u^{\prime}, r-r^{\prime}\right)=\frac{I_{0}\left(r r^{\prime} / 2\left(u-u^{\prime}\right)\right)}{2\left(u-u^{\prime}\right)} \mathrm{e}^{-\left(r^{2}+r^{\prime 2}\right) / 4\left(u-u^{\prime}\right)} H\left(u-u^{\prime}\right),
$$

where $I_{0}$ is a modified Bessel function. The function $G$ solves the heat equation in a cylinder

$$
\frac{\partial}{\partial u} G-\frac{1}{r} \frac{\partial}{\partial r} r \frac{\partial}{\partial r} G=\delta\left(u-u^{\prime}\right) \frac{1}{r} \delta\left(r-r^{\prime}\right) .
$$

Using Eq.(35), it is possible to estimate the damping by $\alpha$-particles born on different flux surfaces. In a tokamak, the concentration of $\alpha$-particle births is largest in the center, where the percentage of energetic $\alpha$-particles would be correspondingly largest too. The $\alpha$-particles would diffuse away from the center as they slowed down. Their contribution to wave-damping off-center would then be as less energetic particles, so that the wave-particle resonance is more easily circumvented.

The key simplifying assumption in deriving this Green's function is in the assumed form of the $\alpha$-particle transport, especially in the assumption that the radial diffusion coefficient depends on $v_{\perp}$ only. This assumption completely decouples the parallel from the perpendicular velocity space dynamics, allowing us to integrate Eq.(28) over the parallel direction to find an equation for $F\left(v_{\perp}, r, t\right)$. In the absence of this decoupling, such a large simplification appears unlikely, and although a Green's function still exists (since the equation is linear), it is not likely to be so simple.

To examine a special case of particular interest, suppose the $\alpha$-particles are all born on the axis, $r=0$, i.e.,

$$
\dot{n}_{s}(r)=\frac{\dot{N}}{2 \pi r} \delta(r),
$$

then

$$
\Psi(r, u)=\dot{N} \int_{u_{\alpha}}^{u} d u^{\prime} \frac{\mathrm{e}^{-r^{2} / 4\left(u-u^{\prime}\right)}}{4 \pi\left(u-u^{\prime}\right)} S\left(u^{\prime}\right),
$$

where $u_{\alpha} \equiv u\left(v_{\alpha}\right)$. Note that we have defined $u$ to be negative, and the range of interest is $0>u>u_{\alpha}$. 
Suppose, further, the special case of a radial diffusion coefficient of the form, $D_{r}\left(v_{\perp}\right)=$ $\tau_{\alpha} v_{\perp}^{2}$. Then we can perform tine integral in Eq.(36). First, note that from Eq.(31),

$$
u\left(v_{\perp}\right)=-\frac{\tau_{\alpha}}{\nu} \int_{0}^{v_{\perp}} v_{\perp} d v_{\perp}=-\frac{\tau_{\alpha}}{2 \nu} v_{\perp}^{2}
$$

so that the source $S$ becomes

$$
S(u)=\frac{1}{2 \pi v_{\alpha} \sqrt{2 \nu \tau_{\alpha}}}\left(u-u_{\alpha}\right)^{-1 / 2}
$$

Substituting now for the source in Eq.(36), we have

$$
\begin{aligned}
\Psi(r, u) & =\frac{1}{4 \pi^{2} v_{\alpha} \sqrt{2 \nu \tau_{\alpha}}} \int_{u_{\alpha}}^{u} \frac{e^{-r^{2} / 4\left(u-u^{\prime}\right)}}{2\left(u-u^{\prime}\right)}\left(u^{\prime}-u_{\alpha}\right)^{-1 / 2} d u^{\prime} \\
& =\frac{1}{4 \pi^{2} v_{\alpha} \sqrt{2 \nu \tau_{\alpha}}} \int_{0}^{u-u_{\alpha}} \frac{\mathrm{e}^{-r^{2} / 4 x}}{2 x}\left(u-u_{\alpha}-x\right)^{-1 / 2} d x \\
& =\frac{\Gamma(1 / 2)}{8 \pi^{2} v_{\alpha} \sqrt{2 \nu \tau_{\alpha} \pi\left(u-u_{\alpha}\right)}} \mathrm{e}^{-r^{2} / 8\left(u-u_{\alpha}\right)} K_{0}\left(\frac{r^{2}}{8\left(u-u_{\alpha}\right)}\right)
\end{aligned}
$$

where $K_{0}$ is a modified Bessel function.

From Eq.(39), the conclusion can be drawn that there is damping but never amplification of the wave, both near and far from the source at the axis. This can be seen by substituting for $u$ and $\Psi$ to get

$$
i\left(v_{\perp}, r\right)=\dot{N} \frac{\Gamma(1 / 2)}{8 \pi^{2} \tau_{\alpha} v_{\alpha} v_{\perp}^{2} \sqrt{\pi\left(v_{\alpha}^{2}-v_{\perp}^{2}\right)}} \mathrm{e}^{-r^{2} / 4 \frac{\tau_{\alpha}}{\nu}\left(v_{\alpha}^{2}-v_{\perp}^{2}\right)} K_{0}\left(\frac{r^{2}}{4 \frac{\tau_{\alpha}}{\nu}\left(v_{\alpha}^{2}-v_{\perp}^{2}\right)}\right) .
$$

In this case, there is clearly no wave growth since $F\left(v_{\perp}, r\right)$ is a monotonically decreasing function of $v_{\perp}$ for all $r$.

A very interesting qrestion is what diffusion coefficient can result in local amplification of the wave. In other words, although the spatially inte rated distribution function is monotonically decreasing, hence Landau damping, there mi y be radii at which the local distribution function is inverted and hence Lan'.du amplifyi.ts It would appear that if high- $v_{\perp}$ but low $v_{\|} \alpha$-particles diffuse the most quickly, then there may be an inversion in $v_{\perp}$-space at large radial distances from the source. Such a diffusion coefficient actually violates the condition under which Eq.(39) was derived, but one might also suppose that the parallel dependency here is inconsequential. It turns out that finding the class of diffusion coefficients that allow or disallow growth is more challenging than one might have expected, and we must leave as an open question such proofs for all but the simple case we considered, namely, a radial diffusion coefficient of the form, $D_{r}\left(v_{\perp}\right)=\tau_{\alpha} v_{\perp}^{2}$.

\section{Nonlocal Current Drive}

If $\alpha$-particle damping can be avoided through off-center deposition of the lower hybrid power, then the critical question becomes whether the superthermal current carriers might 
diffuse to the plasma center before slowing down. Should that happen, the plasma current could appear on magnetic surfaces far from the surface of power deposition. Initially, there would be an induced electric field that opposes the production of current anywhere the current appears, but, eventually, there will evolve a steady state in which there is no electric field, and the plasma current will follow the profile of the diffusing superthermal electrons.

Does this nonlocal current drive occur? The consequences of such a diffusive process have largely been imagined to be negative. That would be true, for example, if the diffusion led to severe power loss, or if the diffusion were so powerful that current carriers were expelled from the tokamak before any current drive effect were realized. However, for our purposes, if such a diffusion were moderate, the consequences could be quite beneficial in allowing the full current to be driven by lower-hybrid waves. While a number of physical mechanisms for fast electron diffusion have been proposed, ${ }^{15-16}$ and the mathematical formalism of nonlocal current drive has been worked out, ${ }^{17}$ what is lacking is an empirical estimate of the diffusion coefficient.

The most detailed evidence, at present, comes from the PLT (Princeton Large Torus) current-drive and ramp-up experiments. ${ }^{18}$ In the ramp-up experiments, in which the current is rising due to the $\mathrm{rf}$ waves, as much as $40 \%$ of the if power was converted into poloidal field energy. The fact that the energy conversion can be so efficient, something that is consistent with the theory, ${ }^{19-20}$ is strong evidence for the model of classical electron collisions on which the theory is based. These experiments spanned several parameter regimes, leading to different physics regimes too, including that of steady-state current drive, ramp-up of the current, and even the unsuccessful sustainment of the current.

In the interpretation of PLT experiments, an attempt was made to check the theory of the electron dynamics without making many assumptions concerning the details of either the theory of wave propagation or wave damping. ${ }^{21}$ This was accomplished by comparing dimensionless quantities each of which depended upon the wave being absorbed. Over 250 shots were tabulated, and with essentially only one free adjustable parameter, namely how much of power could be absorbed, the fit to the theoretical predictions was remarkable. The second implication concerns confinement of the fast electrons.

That the current drive and current ramp-up effects were observed so clearly indicates that the fast electrons must be reasonably well confined, at least on the order of their collision time. Otherwise the current-drive effect is significantly diminished. ${ }^{22}$ These experiments also give an upper bound to the confinement time of these electrons, because of the very high efficiency of the energy conversion. The conversion of wave energy to poloidal field nergy is efficient only if the rf works primarily against an opposing electric field ratiner than against collisions. Such a strong elect ic field will produce energetic runaway electrons, and because of the of waves, the number of runaways is substantial. Since the runaway electrons convert the field energy back to electron kinetic energy, even a small number of runaways ruins the ramp-up effect. Hence, the the observed data implies strongly that these runaway electrons could nui be long confined.

Rough bounds can then be placed on the confinement time $\tau_{\text {conf }}(\mathbf{v})$ of the fast elec- 
trons, based on the PLT data.

$$
\frac{\tau_{c}(\mathbf{v})}{R(\mathbf{v})}>\tau_{\text {conf }}(\mathbf{v})>\tau_{c}(\mathbf{v})
$$

where $\tau_{c}(\mathbf{v})$ is the slowing down time of an electron initially with velocity $v$. The second inequality states that electrons are confined long enough to be slowed down by collisions, something necessary for the current-drive effect. The first inequality states that the effect of runaway electron energy drain is less important than the effect of resonant electrons, somethir.g necessary for the current ramp-up effect. Every resonant electron supports the current ramp-up for a slowing down time, but works against it, with about the same efficacy, for a confinement time in the event that it becomes a backwards runaway, an event occurring with probability $R(\mathbf{v}) \cdot{ }^{20}$ Typical slowing down times in these experiments were on the order of ten milliseconds and typical runaway probabilities in the resonant region were on the order of 0.1 .

Consistent with the moderate diffusion coefficients inferred from the PLT data are very recent experiments on JET and Tore Supra. ${ }^{23-24}$ Here the inference of a moderately large diffusion coefficient was reached by comparing soft $x$-ray and hard $x$-ray measurements.

\section{Conclusions}

Several issues have become apparent in the effort to implement in tokamak reactors current drive by lower hybrid waves. In this work we have expanded upon the key issues related to the $\alpha$-particle environment. We wish, in this section, to summarize our findings, and to address what further experimental effort might point to circumventing the problems that we have outlined.

As discussed by several authors, the damping by $\alpha$-paticles is significant enough to prevent efficient current drive in the plasma center. In Secs. 2 and 3 we supported the conclusions of these authors with an analytic calculation of power dissipation that is both precise and simple. Our observation was that the problem of $\alpha$-particle damping could actually be posed precisely over the region of interest in tokamaks as a one-dimensional problem in velocity space. In addition we derived a $4 / 5$ law for power dissipation in the asymptotic limit of high power waves.

Given the necessity for avoiding $\alpha$-particles at any power, what is first needed is an account of the $\alpha$-particle distribution as a function of both minor radius and velocity. As energetic $\alpha$-particles diffuse from the plasma center, we can expect them to also to slow down, which alleviates the requirement on the lower hybrid wave phase velocity. In addition, away from the tokamak center, the plasma is less dense, so the perpendicular lower hybrid wave phase velocity is in any event faster and more likely to avoid the $\alpha$ particles. It may also be the case that the $\alpha$-particle distribution as a function of radius could develop anisotropies in velocity space. That would mean that, in principle, there could be local amplification of the lower hybrid waves.

In Sec. 4, we derived an integral equation for the $\alpha$-particle distribution for a certain class of spatial diffusion. In Sec. 5, we argued that there is a possibility too of spatial diffusion of lower hybrid current carriers that might produce current in the tokamak center with power dissipated off-center. 
What is needed, however, is firmer empirical evidence of both the radial transport of the fast electrons and the radial transport of the confined $\alpha$-particle as they slow down. Also, because of the critical role played by the wave phase velocity, which governs the resonant interaction, a more precise study of the perpendicular index of refraction in toroidal geometry may be useful. Only with such empirical evidence can we evaluate the possibility of maintaining current drive by lower hybrid waves in a tokamak D-T reactor.

What are the necessary experiments? The physics of $\alpha$-particle transport is under study for other reasons, particularly energy confinement. Although our problem presents some peculiarities, in particular with respect to anisotropic distributions, the physics of $\alpha$-particle transport may have to await burning experiments. On the other hand, probably a great deal more effort could be made to understand the physics of fast electron transport. In Sec. 5, we tried to infer bounds on the radial transport of these electrons through global considerations, such as the rate of current production.

An alternative that may give more precise information on electron transport would be to measure with radial resolution signatures of the fast electrons, such as the synchrotron radiation ${ }^{25-26}$ or the bremsstrahlung. Such measurements would reveal more if at the same time an effort were made to localize in space, and possibly in time, the power dissipation. The advantage of localizing in time is that the transport process might be followed directly if time-resolved measurements can be made. A second advantage is that in the absence of spatial resolution, it might be possible to infer from the time-resolved measurements certain plasma parameters, including those governing the spatial transport. One consideration in localizing in time the power deposition is that the transport itself may be affected by the concommitant induced electric field. Under steady power conditions, however, a steady state is achievable with non-local current production exactly in the same way as with local production.

In summary, a number of concrete issues need to be clarified before definitive statements can be made on using lower hybrid current drive to provide all the required noninductive current in a D-T tokamak reactor. 'The most likely possibility for efficient current production by this means probably would rely upon the deposition of lower hybrid power somewhat off-center, with the subsequent penetration of the plasma center by the fast, superthermal electrons.

\section{Acknowledgments}

The authors would like to thank Dr. William $\mathrm{N}$ ?vins for a number of very stimulating conversations. This work was supported by the United States Department of Energy under contract number DE-AC02-76-CHO3073.

\section{References}

[1] FisCh N. J., Phys. Rev. Lett. 59 (1978) 175.

[2] Takase Y., Knowlton S., and Porkolab M. (1987) Phys. Fluids 30, 1169.

[3] Bernabei S., et al., Phys. Rev. Lett. 49 (1982) 1255.

[4] Leuterer F., Eckhartt D., Söldner F., Becker G., Brambilla M., Brinkschulte H., Derfler H., Ditte U., Eberhagen A., Fussmann G., Gehre O., Gernhardt J., Gierke G. v., Glock E., Gruber O., Hass G., Hesse M., 
Janeschitz G., Karger F., Keilhacker M., Kissel S., Klüber O., Kornherr M., Lisitano G., Magne R., Mayer H. M., McCormick K., Meisel D., Mertens V., Müller E. R., Münich M., Murmann H., Poschenrieder W., RapP H., Ryter F., Schmitter K. H., Schneider F., Siller G., Smeulders P., Steuer K. H., Vien T., Wagner F., Woyna F. v., Zouhar M., Phys. Rev. Lett. 55 (1985) 75.

[5] Maekawa T., Saito T.,Nakamura M., Cho T.,Kubo S.,Shimozuma T., Terumichi Y.,Hamada Y.,Tanaka S., Phys. Lett. A 85 (1981) 339.

[6] Wong, K. M., ONo, M., Nucl. Fusion 24 (1984) 615.

[7] Bonoli, P. T., Porkolab M. (1987), Nucl. Fusion 27, 1347.

[8] Barbato, E., Santini, F., Nucl. Fusion 31 (1991) 673.

[9] Spada, M., Bornatici, M., Engelmann, F., Nucl. Fusion 31 (1991) 447.

[10] Nevins, W., personal communication.

[11] Karney, C. F. F., Phys. Fluids 22 (1979) 2188.

[12] White, R. B., Mynick, H. E., Phys. Fluids B 1 (1989) 980.

[13] Goloborod'ko, V. Ya., Kolesnichenko, Ya. I., YaVorskiJ, V. A., Physica Scripta T16 (1986) ppp.

[14] Chen, L., Vaclavik, J., Hammett, G. W., Nucl. Fusion 28 (1988) 389.

[15] Rechester, A., Rosenbluth, M., Phys. Rev. Lett. 40 (1978) 138.

[16] Stix, T., Nucl. Fusion 18 (1978) 353.

[17] Rax J. M., Moreau, D., Nucl. Fusion 29 (1989) 1751.

[18] Jobes F. C., Bernabei S., Chu T. K., Hooke W. M., Merservey E. B., MotLey R. W., Stevens J. E., and von Goeler S., Phys. Rev. Lett. 55 (1985) 1295.

[19] Fisch N. J., Karney C. F. F., Phys. Rev. Lett. 54 (1985) 897.

[20] FisCh N. J., Rev. Mod. Phys. 59 (1987) 175.

[21] Karney C. F. F., Fisch N. J., Jobes F. C., Phys. Rev. A 32 (1985) 2554.

[22] LuCKhaRdT S. C. (1987) Nucl. Fusion 27, 1914.

[23] Gormezano C. et al., Proc. 18th European Physical Society Conf. on Controlled Fusion and Plasma Physics (Berlin, 1991) III-393.

[24] Moreau D. et al., Proc. 18th European Physical Society Conf. on Controlled Fusion and Plasma Physics (Berlin, 1991), to appear in Plasma Physics and Controlled Fusion.

[25] Luce T., Efthimion P., and Fisch N. J. (1988) Rev. Sci. Instr. 59, 1593.

[26] FisCh N. J., Plasma Physics and Controlled Fusion 30 (1988) 1059. 
Dr. F. Paoloni, Univ. of Wollongong, AUSTRALIA

Prot. M.H. Brennen, Univ. of Sydnoy, AUSTRALIA

Plasma Research Lab., Australian Nat. Univ., AUSTRALIA

Prof. I.R. Jones, Flinders Univ, AUSTRALIA

Prot. F. Cap, Inst. for Theoretical Physics, AUSTAIA

Prof. M. Heindler, Institut for Theoretische Physik. AUSTRIA

Prot. M. Goossens, Astronomisch Instituut, BELGIUM

Ecole Royale Militaire, Lab. de Phy. Plasunas, BELGIUM

Commission-Europeen, DG. XII-Fusion Prog., BELGIUM

Prof. R. Bouciqie, Rijksuniversiteit Gent, BELGIUM

Dr. P.H. Sakanaka, Instituro Fisica, BRAZIL

Instituto Do Pesquisas Especiais-INPE, BRAZIL

Documents Office. Abmic Eneroy of Canada Lid., CANADA

Dr. M.P. Bachynaki, MPB Tectnologios, Inc. CANADA

Dr. H.M. Skeregerd, Univ. of Seskatchewan, CANADA

Prof. J. Teichmenn, Univ. of Mrmtreal, CANADA

Prot. S.R. Sreenivasan, Univ. of Calgary, CANADA

Prof. T.W. Johnston, INRS-Energie, CANADA

Dr. R. Bolton, Contre canadien de fusion magnbique, CANADA

Dr. C.R. Jemes., Univ. of Aberte. CANADA

Dr. P. Lukse, Komenskeho Universzita, CZECHOSLOVAKIA

The Librarian, Cuthem Laborabry, ENGLAND

Librey, R61, Ruthertord Appleton Laboratory, ENGLAND

Mrs. S.A. Murctinson, JET Library, ENGLAND

P. Muconen. Univ. of Heleinto, FINLAND

C. Mouteot, Lab. do Physique des Milieux lonisés, FRANCE

J. Radot, CEN/CADARACHE - Bat 506, FRANCE

Me. C. Rinni, Univ. of loannina, GREECE

Dr. T. Mul, Acaderny Bibliographic Ser., HONG KONG

Preprint Library. Hungarian Academy of Sai., HUNGARY

Dr. B. Das Gupten Saha Inst. of Nuclear Physics. INDIA

Dr. P. Kaw, Inst. for Plesma Rocearch, INDIA

Dr. P. Rosenau, lareel Inat of Tectnology, ISRAEL

Librarian, Intomationa Conter for Theo Physics, ITALY

Miss C. Do Palo, Associazione EURATOM-ENEA, ITALY

Dr. G. Groseo, Istituto di Fisica dol Plasma, ITALY

Dr. H. Yamab, Toshiba Res a Dovel Centor. JAPAN

Prof. I. Kawakami, Abomic Energy Ros.Inst, JAPAN

Prot. K. Nishikewa, Hiroshima Univ., JAPAN
Director, Japan Atomic Energy Research Inst., JAPAN

Prot. S. Itoh, Kyushu Univ., JAPAN

Data and Planning Conter, Negoya Univ., JAPAî́

Prof. S. Tanaka, Kyoto Univ., JAPAN

Library. K) oto Univ., JAPAN

Prot. N. Inoue. Univ. of Tokyo. JAPAiN

S. Mori, Tectnical Advisor, JAERI, JAPAN

O. Mitarai, Kumamoto Inst, of Tectnology. JAPAN

H. Jeong. Korea Advanced Energy Research inst., KOREA

Prof. D.I. Choi, The Korea Adv. Inst. of Sa. \& Tech., KOREA

Prof. B.S. Liby, Univ. of Waiketo. NEW ZEALAND

Inst. of Plasma Physics. PEOPLE'S REPUBLIC OF CHINA

Librarien, Inst of Physics, PEOPLE'S REPUBLC OF CHINA

Library. Tringhua Univ., PEOPLE'S REPUBLIC OF CHINA

2. L. S.W. Inst Physica, PEOPLES REPUBUC OF CHINA

Prof. J.A.C. Cabrd, Instivis Superior Tecrico, PORTUGAL

Dr. O. Potrus, AL I CUzA Univ., ROMANIA

Dr. J. of Villiers, Fusion Sudies, AEC. S. AFRICA

Prof. M.A. Helboro. Univ. of Natal, S. AFAiCA

C.I.E.MA.T, Fusion Divition Librery, SPAIN

Dr. L Sionflo, Univ. of UMEA, SWEDEN

Librey, Poyed Inst of Technology, SWEDEN

Prof. H. Wilhermson, Chalmers Univ. of Tech., SWEDEN

Centre Phys. Des Plasmas, Ecole Potytoch, SWIZERLAND

Bibliothook, Inst. Voor Plasma-Fyeica. THE NETHERLANDS

M. Durgut, Vice Chaimen, Niddlo East Tech. Univ., TURKEY

Dr. D.D. Ayutov, Siberian Branch of Acaderny of Sa., USSR

Dr. G.A. Eliseov, Kurchabov Inst, USSR

Librerien. The Ukr.SSR Academy of Scioncos. USSR

Dr. L.M. Kovrizhnykh, Inst of Generd Physics, USSR

Kemfor:zinngeanlege GmbH, Zentrabibtiothek. W. GERMANY

Bibliothok, Inst. For Plasmatorschung. W. GERMANY

Prof. K. Schinder, Ruhr-Universittl Bochum, W. GERMANY

Or. F. Wagner, (ASOEX), Max-Planck-Institut, W. GERMANY

Librerian, Max-Planck-Institut, W. GERMANY

Prot. R.K. Janov, Inst. of Physics, YUGOSLAVIA 

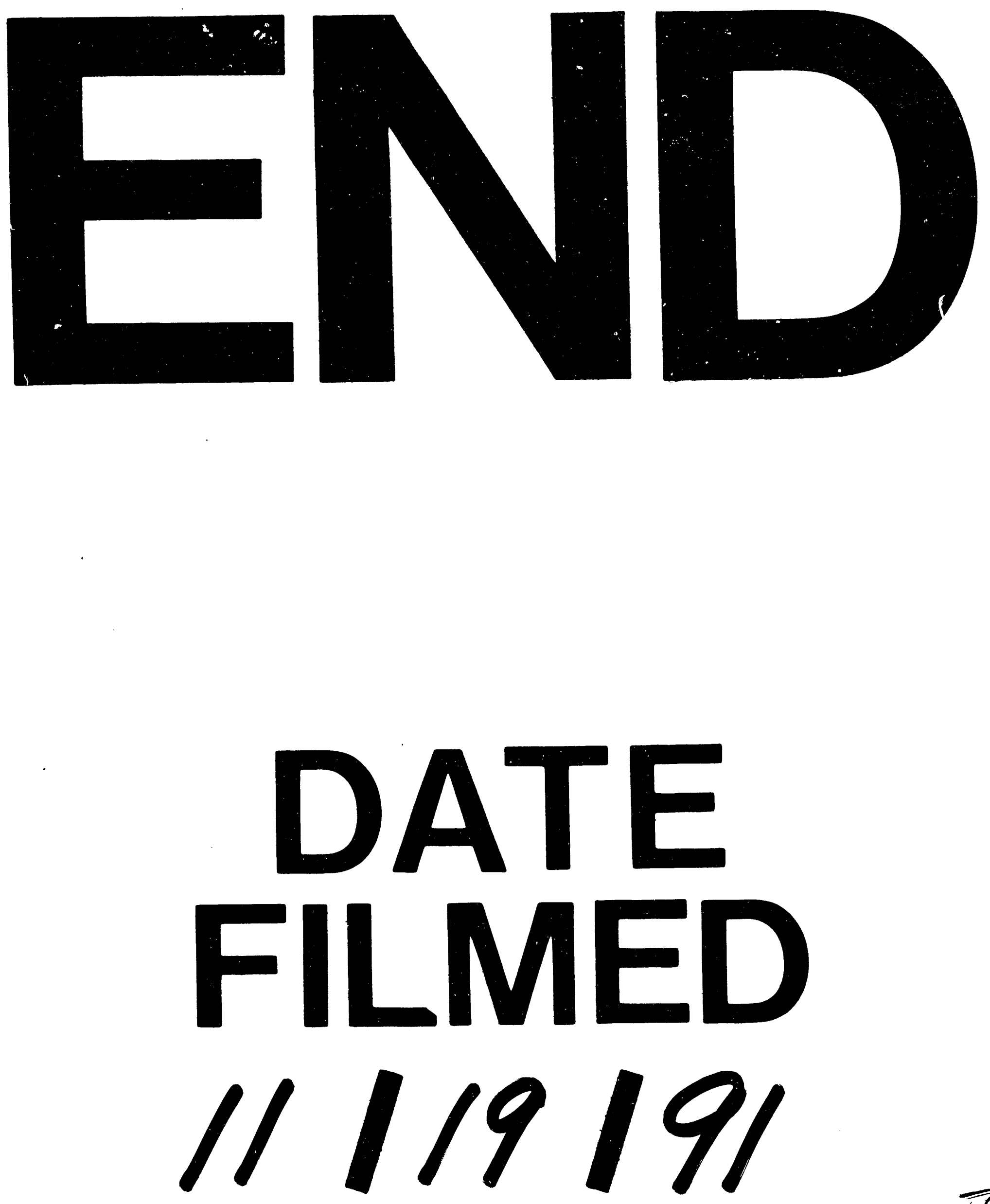

I 
